\title{
Instrucción en estrategias de comprensión lectora mediante enseñanza recíproca: efectos del agrupamiento de los estudiantes
}

\author{
Manuel Soriano-Ferrer ${ }^{1 *}$, Pilar Sánchez-López ${ }^{2}$, Encarnación Soriano-Ayala ${ }^{3}$ y Francisco Nievas-Cazorla ${ }^{2}$ \\ 1 Departamento de Psicología Evolutiva y de la Educación. Universidad de Valencia. \\ 2 Departamento de Psicología Evolutiva y de la Educación. Universidad de Almería. \\ 3 Departamento de Métodos de Investigación y Diagnóstico en Educación. Universidad de Almería
}

\begin{abstract}
Resumen: El objetivo de este estudio es comparar el efecto que dos formatos de agrupamiento de los estudiantes tiene sobre la comprensión lectora. La instrucción en comprensión lectora empleó la Enseñanza Recíproca (ER) que es un procedimiento que enseña a los estudiantes estrategias cognitivas para incrementar la comprensión lectora. Participaron 43 alumnos de $4^{\circ}$ de E.P.: 18 fueron instruidos mediante ER en el aula ordinaria (GG); 8 mediante ER en pequeño grupo (PG) y 17 recibieron la enseñanza tradicional. Se emplearon dos tipos de medidas: medidas de efectos específicos (idea principal y supervisión y regulación) y medidas de generalización (test estandarizado, significado implícito y recuerdo). Los resultados demuestran que los dos grupos instruidos mediante ER fueron superiores en las medidas de efectos específicos y en las de generalización al grupo de comparación. Además, el grupo instruido en pequeño grupo (PG) fue superior al GG y GC en la mayoría de las medidas de efectos específicos y de generalización.
\end{abstract}

Palabras clave: Enseñanza recíproca; comprensión lectora; modalidad de agrupamiento; instrucción.

\begin{abstract}
Title: Instruction of Reading Comprehension Strategies through Reciprocal Teaching: Effects of Grouping Formats.

Abstract: The aim of this study was to compare the effects of two grouping formats on the reading comprehension. Reading comprehension Instruction used reciprocal teaching (RT) that is an instructional procedure to teach students cognitive strategies that might lead to improved reading comprehension. A sample of 43 students in the fourth year of primary education was selected: 18 children were instructed in regular classroom (GG), 8 were instructed in small group (PG), while the remaining 17 made up the comparison group (GC). Two types of comprehension measures were used: tasks of specific effects (getting the main idea, comprehensionmonitoring test) and transfer effect measures (standardized test, word meaning inference, and free recall). Thus, the results show that both RT conditions benefited from the instruction, performing better than the comparison group in measures of specific effects and in the transfer effect measures. Furthermore, students from small group (PG) outperformed students in GG and GC on specific effects and in the transfer effect measures.
\end{abstract}

Key words: Reciprocal teaching; reading comprehension; grouping formats; instruction.

\section{Introducción}

El logro de una buena comprensión lectora es, y ha sido, una de las principales metas de la enseñanza primaria, debido a que sobre la comprensión lectora se asienta una gran parte de los aprendizajes de la enseñanza secundaria (Alvermann y Earle, 2003; Johnston, Barnes y Desrochers, 2008; Madariaga y Martínez, 2010; Spörer, Brunstein y Kieschke, 2009; Vaughn y Klingner, 2004). Además, diferentes estudios observacionales han mostrado que los profesores no suelen emplear procedimientos de instrucción de la comprensión lectora (Durkin, 1978-79; Ness, 2008; Solé, 1987; Solé y Teberosky, 2002).

Por ello, no resulta extraño que durante los últimos años se hayan realizado diferentes revisiones acerca de los procedimientos más efectivos para mejorar la comprensión lectora (Berkeley, Scruggs, y Mastropieri, 2010; Dole, Nokes, y Drits, 2009; Jitendra, Burgess, y Gajria, 2011; National Reading Panel, 2000; Raphael, George, Weber, y Nies, 2009; Sencibaugh, 2007; Solis et al., 2011). En términos generales, la investigación sobre la mejora de la comprensión lectora se ha centrado en la enseñanza de las estrategias de comprensión que utilizan los buenos comprendedores. Aunque la mayoría de los trabajos han instruido a los estudiantes en estrategias aisladas de comprensión, algunos han empleado la

* Dirección para correspondencia [Correspondence address]: Manuel Soriano Ferrer. Departamento de Psicología Evolutiva y de la Educación. Facultad de Psicología. Avda. Blasco Ibañez, 21. 46010 Valencia (España). E-mail: Manuel.Soriano@uv.es enseñanza conjunta de múltiples estrategias de comprensión, como en el procedimiento conocido con el nombre de enseñanza recíproca (Palincsar y Brown, 1984; Brown y Palincsar, 1989).

La Enseñanza Recíproca (ER) se caracteriza por "la práctica guiada en la aplicación de estrategias simples y concretas en la tarea de comprensión de un texto" (Brown y Palincsar, 1989, pp. 413): predecir el contenido del texto en los siguientes párrafos, clarificar las dificultades que el texto presenta, generar preguntas sobre el texto y resumir. Las autoras escogieron estas cuatro estrategias porque cumplían una doble función: incitaban a los chicos a comprender aquello que leían y, al mismo tiempo, proporcionaban una oportunidad al estudiante para comprobar si realmente estaba comprendiendo. Como en todas las teorías socioconstructivistas, en este procedimiento se asume que a través del dialogo profesor-alumno y alumno-alumno los estudiantes internalizan y generalizan el uso de esas cuatro estrategias, aprendiendo a supervisar y regular su propia comprensión (Palincsar y Klenk, 1992; Brown y Campione, 1998). Además, en este proceso de internalización de las estrategias, es fundamental el andamiaje del profesor, ya que, los niños primero experimentan el conjunto de actividades cognitivas en presencia de expertos, y sólo gradualmente ejecutan estas funciones por sí mismos, de forma que la responsabilidad de las actividades de comprensión es transferida a los estudiantes tan pronto como es posible, con una ayuda ajustada a sus necesidades. Es decir, se trata de promover y provocar que los alumnos dirijan su propio aprendizaje a partir de los textos (Brown, 1997; Brown y Campione, 1998; Palincsar y Klenk, 1992). Es 
decir, primero un experto guía la actividad del niño haciendo la mayor parte del trabajo, luego conforme el niño se hace más experimentado y capaz de ejecutar aspectos más complejos de la tarea, el adulto gradualmente le cede mayor responsabilidad. Así, el adulto y el niño se dividen el trabajo cognitivo, con el niño tomando la iniciativa y el adulto corrigiendo y guiando la realización de la tarea. Finalmente, el adulto permite al niño encargarse del papel principal y adopta una posición de espectador (Brown, 1997; Brown y Campione, 1998; Hacker y Tenent, 2002; Mason, 2004).

La efectividad de la ER ha sido demostrada con estudiantes de diferentes edades, con y sin dificultades de comprensión, especialmente cuando ha sido implementada bajo la supervisión de expertos (Alfassi, 1998, 2004; Brown y Palincsar, 1989; Garjiria, Jitendra, Sood y Sacks, 2007; Hacker y Tenent, 2002; Mason, 2004; Rosenshine y Meister, 1994; Seymour y Osana, 2003; Spörer, Brunstein, y Kieschke, 2009). Respecto a la eficacia de la ER, uno de los resultados más sorprendentes de la revisión de 16 estudios realizada por Rosenshine y Meister (1994) es su mayor efectividad cuando se emplean medidas de comprensión desarrolladas por los investigadores (tamaño del efecto .88) que cuando se emplean test estandarizados de comprensión (tamaño del efecto .32).

Partiendo de estas consideraciones, en el presente trabajo nos planteamos dos objetivos. Primero, comprobar la eficacia que la ER aplicada en aulas ordinarias por su profesor tiene para enseñar estrategias de comprensión. En este entrenamiento empleamos la ER con enseñanza explícita de las estrategias de comprensión, puesto que de esta forma se optimizan los resultados, ya que ha mostrado ser más efectiva cuando se emplea otro procedimiento previo de enseñanza de las estrategias (Rosenshine y Meister, 1994). Segundo, comparar la eficacia de la instrucción en estrategias de comprensión mediante enseñanza recíproca desarrollada en pequeño grupo con la instrucción de dichas estrategias en gran grupo. Esto es, determinar si la modalidad de agrupamiento de los alumnos (pequeño versus gran grupo) incide en la eficacia de la enseñanza recíproca. La ER se ha empleado con grupos de instrucción que han oscilado entre 2 y 23 alumnos. Sin embargo, ningún estudio ha analizado si la modalidad de agrupamiento de los alumnos incidía en las ganancias en comprensión lectora empleando ER, a pesar de que la eficacia de los programas de intervención se ve influida por la modalidad de agrupamiento de los estudiantes (O'Connor, 2000; Pikulski, 1991; Moody, Vaughn, y Schumm, 1997). En este sentido, el meta-análisis de los 30 trabajos publicados entre 1975 y 1995 desarrollado por El- baum, Vaughn, Hughes y Moody (1999) muestra claramente la superioridad del pequeño grupo sobre la instrucción desarrollada en el aula entera sobre los logros en lectura. Por ello, esperamos que las ganancias en las medidas de comprensión sean superiores en el grupo de niños que han sido entrenados en pequeño grupo mediante ER.

\section{Método}

\section{Sujetos}

Los 58 alumnos que participaron en esta investigación estaban cursando cuarto curso de Enseñanza Primaria y pertenecían a tres aulas del mismo centro privado. Fueron eliminados de la muestra 15 alumnos, debido a que no habían asistido a alguna de las sesiones en las que se desarrolló la intervención, o a alguna en las que se aplicaron los instrumentos de evaluación. En consecuencia, la muestra final estuvo compuesta por 43 alumnos con una media de 9 años y 3 meses, de los cuales el $51.2 \%$ eran varones y el $48.8 \%$ mujeres. De éstos, 18 pertenecían al grupo experimental 1, que recibieron instrucción en estrategias de comprensión y aprendizaje a partir de textos mediante enseñanza recíproca en gran grupo (GG), 8 pertenecían al grupo experimental 2, que recibió instrucción en estrategias de comprensión mediante enseñanza recíproca en pequeño grupo (PG), y finalmente, 17 se emplearon como grupo de comparación (GC)(véase Tabla 1).

Debido a que se emplearon aulas naturales y los sujetos no pudieron ser asignados de forma aleatoria a las condiciones experimentales, se comprobó que no existiesen diferencias de partida en edad, inteligencia y en comprensión de textos antes de iniciar las intervenciones. Para evaluar la inteligencia no verbal se administró la Escala 2 (forma A) del Factor "g" de Cattell y Cattell (1950/1989). Para evaluar la comprensión se emplearon dos tareas. Una de ellas es la subprueba de comprensión lectora de las Pruebas Psicopedagógicas de Aprendizajes Instrumentales (Canals, 1988) que consta de diferentes tareas: ordenación de frases, seguimiento de instrucciones, identificación de idea principal y contestar preguntas a un texto expositivo. La otra fue la subprueba de comprensión de textos del PROLEC (Cuetos, Rodríguez y Ruano, 1996). En esta tarea el niño debe contestar a preguntas literales e inferenciales acerca de cuatro textos, dos narrativos y dos expositivos. En ambas pruebas se emplearon los criterios de corrección del manual.

Tabla 1. Media y (Desviación Típica) de edad, inteligencia y comprensión de textos.

\begin{tabular}{lccc}
\hline & \multicolumn{1}{c}{ Gran Grupo $(\mathrm{GG} ; n=18)$} & Pequeño Grupo (PG; $n=8)$ & Grupo Comparación $(\mathrm{GC} ; n=17)$ \\
\cline { 2 - 4 } & $M(\mathrm{DT})$ & $M(\mathrm{DT})$ & $M(\mathrm{DT})$ \\
\hline Edad & $9.28(0.46)$ & $9.38(0.51)$ & $9.41(0.5)$ \\
Inteligencia & $114.5(13.36)$ & $106.13(12.1)$ & $109.29(11.46)$ \\
Comprensión Canals & $5.33(0.78)$ & $5.12(0.83)$ & $5.5(1.01)$ \\
Comprensión Prolec & $12(1.32)$ & $12(1.6)$ & $12.23(1.6)$ \\
\hline
\end{tabular}


Tras comprobar que se cumplía el criterio de normalidad estadística, se procedió a comparar a los tres grupos, antes de iniciar la intervención, mediante un análisis de varianza (ANOVA) de comparación entre grupos. Tal y como se puede comprobar en la Tabla 1 , los resultados de los análisis estadísticos realizados en el pretest indicaron que no se encuentran diferencias significativas entre los sujetos de los tres grupos en ninguna de las variables: edad, $F_{(2,40)}=.34, p$

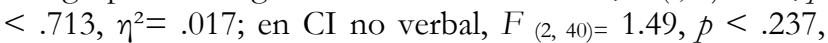
$\eta^{2}=.069$; ni en comprensión del Canals, $F_{(2,40)}=.693$, $p<$. $506, \eta^{2}=.033$, o del PROLEC, $F_{(2,40)}=1.28, p<.880, \eta^{2}=$ .006 , lo que indica la igualdad de los grupos antes de iniciar la intervención.

\section{Instrumentos de Evaluación}

Basándonos en las últimas revisiones sobre la evaluación de la comprensión lectora (Afflerbach y Cho, 2011; Leslie y Caldwell, 2009; Stahl, 2009), dos grandes grupos de medidas de comprensión se tomaron en dos momentos, tras la intervención y en el seguimiento (cuatro meses después) para determinar los efectos de los programas de intervención: a) Medidas de Efectos Específicos, constituidas por aquellas habilidades de comprensión instruidas mediante el procedimiento de ER; b) Medidas de Generalización, que hacían referencia a habilidades no instruidas directamente, pero relacionadas con la enseñanza de estrategias de comprensión.

A) Medidas de efectos específicos, compuestas por dos tipos de tareas: (a) Prueba de captación de ideas principales implícitas (Vidal-Abarca, 1989), en la que los niños tenían que escribir la idea principal implícita en tres lecturas de estructura comparativa y enumerativa. En la corrección se otorgaba 1 punto a la respuesta que incluía la idea principal de la lectura, 0.5 a las respuestas que recogían el tema de la lectura pero no la idea principal, y 0 en el caso de cualquier otra respuesta. Se emplearon dos formas paralelas para las fases de postratamiento y de seguimiento respectivamente; (b) Tareas de orden metacognitivo, dirigidas a la evaluación de la supervisión y regulación de la comprensión de textos. Estaba constituida por dos instrumentos: (1) Cuestionario de Estrategias Lectoras (Miranda, Vidal-Abarca y Villaescusa, 1997), con 21 ítems que valoran el conocimiento del niño sobre las estrategias y actividades de comprensión lectora. Se asignó una puntuación de tres (siempre), dos (casi siempre), uno (a veces) y cero (nunca) a cada una de las valoraciones, a excepción de los ítems 3, 4 y 6 que son inversos; (2) Batería SURCO (Alonso Tapia y Carriedo, 1992), de la cual se seleccionaron 12 items con bajo índice de dificultad y alto de homogeneidad, que se distribuyeron en dos pruebas paralelas de 6 ítems que se emplearon en las fases de postratamiento y de seguimiento. Se proporcionan tres índices: detección de fallos de comprensión, conocimiento de estrategias y uso de estrategias para regular la comprensión.

B) Medidas de generalización, compuestas por tres tareas: (a) Prueba estandarizada de comprensión: (1) Subprueba de Com- prensión de Textos de la Batería de Evaluación de la Lectura BEL- (López-Higes, Mayoral y Villoria, 2002), en la que el niño tras la lectura de cada texto, uno narrativo y otro expositivo (nivel 1), debe señalar la respuesta a 12 preguntas; (b) Prueba de Significado Implícito (Gilabert, 1990), que plantea la tarea de inferir el significado de una pseudopalabra a partir de claves contextuales implícitas en seis textos expositivos. Para la puntuación se asignaron 2 puntos si la respuesta contenía la idea completa o su equivalente, 1 punto si contenía la idea pero incluida en un contexto más amplio que no era pertinente o agregaba confusión, y 0 puntos cuando la respuesta no se ajustaba a ninguna de la anteriores categorías. Se emplearon dos formas paralelas para las fases de postratamiento y de seguimiento respectivamente; (c) Prueba de Recuerdo (Soriano, Vidal-Abarca y Miranda, 1996). En la fase de postratamiento, los chicos estudiaron el texto "Los dinosaurios" durante 7 minutos, advirtiéndoseles que deberían recordar posteriormente la información; tras una tarea distractora (5 minutos) se les pidió que escribieran todo aquello que recordaran. Para la puntuación del recuerdo se dividió el texto en ideas-unidad, siguiendo el procedimiento de Mayer (1985), que fueron clasificadas en dos niveles de importancia mediante un sistema de jueces: 12 ideas importantes (nivel 1) y 14 poco importantes (nivel 2). En el texto empleado en la fase de seguimiento "La Contaminación" se identificaron 10 ideas importantes (nivel 1) y 12 ideas poco importantes (nivel 2). Los recuerdos de los niños fueron igualmente divididos en ideas-unidad, otorgándose un punto a cada idea recordada. Tras puntuar independientemente la prueba tres jueces se obtuvo un acuerdo superior al 85\%, resolviendo las discrepancias de común acuerdo.

\section{Programas de intervención}

Los dos grupos de tratamiento recibieron instrucción en estrategias de comprensión mediante ER por su profesor de Conocimiento del Medio, que tenía más de diez años de experiencia. Además, había realizado un curso de doctorado sobre la instrucción de estrategias de comprensión y fue entrenado en un seminario de 6 horas de duración en la instrucción mediante ER. Se mantuvieron 3 entrevistas con el profesor para analizar cómo se desarrollaba el procedimiento de ER. Se optó por realizar el entrenamiento en comprensión a través de su profesor con el fin de que la ER se incorporase al aula de la forma más natural posible, teniendo en cuenta que no se han encontrado diferencias entre los estudios en los que el entrenamiento ha sido realizado por un experimentador o bien por los profesores (Rosenshine y Stevens, 1994).

Inicialmente se explicó a los alumnos que la lectura es un proceso de comunicación en el que el autor intenta transmitir una información que debe ser entendida por el lector. El entrenamiento se desarrolló a lo largo de 50 sesiones, de unos 45 minutos cada una, en dos fases: 1) Enseñanza explíci- 
ta, en la que el profesor modelaba a los niños durante 5 sesiones la aplicación de las cuatro estrategias, más la activación del conocimiento previo. Se utilizaron textos adaptados (Soriano, Vidal-Abarca y Miranda, 1996). Para facilitar el recuerdo de las actividades, el profesor entregaba a cada alumno una tarjeta que recogía dichas actividades; 2) Entrenamiento, que duró 45 sesiones, a razón de tres sesiones semanales. Se emplearon textos naturales expositivos del libro de Conocimiento del Medio, pues, aunque se caracterizan por su contenido interpersonal y su dificultad (De Vega, Cuetos, Domínguez, y Estévez, 1999) son los propios de la enseñanza formal y los que más interesan a los profesores.

Los alumnos del aula completa (gran grupo) fueron asignados a diferentes grupos de discusión (6-8 alumnos), que iban rotando por los grupos para que tuviesen más oportunidades de participar y no asumiesen roles fijos en las diferentes sesiones de instrucción. En cada uno de los grupos un alumno asumía el rol de tutor y dirigía el debate sobre el texto. El procedimiento seguido fue el siguiente: lectura del título del tema, activación de conocimiento previo y predicción del contenido; todos los miembros de cada uno de los grupos leían silenciosamente el primer fragmento del texto y se clarificaban los términos que no se hubiesen entendido, se formulaban preguntas y se resumía el contenido del fragmento leído; el tutor de uno de los grupos, exponía en voz alta el resumen, las preguntas y las respuestas que habían elaborado, así como las predicciones; los tutores de los otros grupos debían dialogar con el que estaba exponiendo, realizando las matizaciones que hubieran surgido en su grupo; al terminar satisfactoriamente con las actividades en el primer fragmento, se pasaba al siguiente hasta finalizar el texto haciendo un resumen global del texto. A lo largo de toda la clase, el profesor ofrecía su guía y retroalimentación de acuerdo con las necesidades y dificultades detectadas.

En el caso de los alumnos entrenados en pequeño grupo (8 alumnos) el procedimiento seguido era idéntico al seguido con los niños entrenados en gran grupo, de forma que la diferencia entre ambas condiciones de instrucción fue la modalidad de agrupamiento de los alumnos, con la salvedad de que el entrenamiento se realizó en horario extraescolar por el mismo profesor.

El grupo de comparación recibió la instrucción ordinaria de su profesor y fue evaluado en el mismo periodo de tiempo y con las mismas medidas que los dos grupos que recibieron entrenamiento en estrategias con enseñanza recíproca.

\section{Análisis de datos}

Para comparar los resultados obtenidos por los distintos grupos se utilizó el análisis de varianza entre grupos (ANO$\mathrm{VA}$ ) en las fases de postratamiento y seguimiento, ya que es una prueba robusta frente al incumplimiento del supuesto de la normalidad, que desafortunadamente ocurre en algunas variables. Sin embargo, hemos decidió adoptar una postura conservadora y ser más exigentes con los niveles de significación a la hora de detectar diferencias entre medias. Así, sólo se aceptarán como significativos los resultados por debajo del 1\%. Posteriormente, se aplicó el test DSH de Tuckey para comprobar las diferencias entre pares de grupos. Además, se aporta el tamaño del efecto estimado a partir de la eta cuadrado $\left(\eta^{2}\right)$.

\section{Resultados}

\section{Resultados obtenidos en las medidas de efectos es- pecíficos (ver Tabla 2)}

Según el ANOVA se encuentran diferencias entre los grupos en relación a la habilidad para captar las ideas principales implícitas de los textos en la fase de postratamiento $\left(F_{(2,40)}=\right.$ 21.975, $\left.p<.000, \eta^{2}=.52\right)$, que se mantienen en la de seguimiento $\left(F_{(2,40)}=5.351, p<.009, \eta^{2}=.21\right)$. El test de Tuckey señala que, tanto en la fase de postratamiento como en la de seguimiento, los dos grupos que recibieron instrucción en estrategias de comprensión aventajaban al grupo de comparación, aunque no se encontraron diferencias entre los grupos instruidos en estrategias de comprensión.

Tabla 2. Medias y desviaciones típicas en las medidas de efectos específicos.

\begin{tabular}{|c|c|c|c|c|c|c|c|}
\hline & & \multicolumn{2}{|c|}{ Gran Grupo (GG; $n=18)$} & \multicolumn{2}{|c|}{ Pequeño Grupo (PG; $n=8)$} & \multicolumn{2}{|c|}{ Grupo Comparación (GC; $n=17)$} \\
\hline & & Postt & Seguim & Postt & Seguim & Postt & Seguim \\
\hline \multirow[t]{2}{*}{ Idea principal implícita } & $M$ & 2.02 & 1.75 & 2 & 1.68 & 0.64 & 0.94 \\
\hline & $D T$ & 0.65 & 0.73 & 0.8 & 0.92 & 0.60 & 0.74 \\
\hline \multirow[t]{2}{*}{ C. Estrategias Lectoras (CEL) } & $M$ & 38.94 & 38.61 & 46.5 & 41.5 & 30.6 & 30.23 \\
\hline & $D T$ & 4.78 & 4.87 & 5.2 & 4.92 & 4.10 & 4.54 \\
\hline \multirow[t]{2}{*}{ Cto. de Estrategias } & $M$ & 3.11 & 3.33 & 3.5 & 4.1 & 1.76 & 2 \\
\hline & $D T$ & 1.07 & 0.97 & 0.53 & 0.64 & 0.9 & 1.21 \\
\hline \multirow[t]{2}{*}{ Uso de Estrategias } & $M$ & 2.16 & 2.38 & 3.1 & 3.6 & 1.3 & 1.36 \\
\hline & $D T$ & 0.61 & 0.77 & 0.64 & 0.51 & 0.86 & 0.90 \\
\hline \multirow[t]{2}{*}{ Detección de Fallos. } & $M$ & 5.05 & 5.5 & 4.12 & 5 & 6.11 & 6.5 \\
\hline & $D T$ & 1.95 & 1.88 & 1.3 & 1.6 & 1.99 & 2.37 \\
\hline
\end{tabular}

El ANOVA entre grupos indica que tras la intervención se encuentran diferencias entre los grupos en el conocimiento de estrategias y actividades de comprensión, tanto en la fase de postra- tamiento $\left(\mathrm{F}_{(2,40)}=34.532, p<.000, \eta^{2}=.63\right)$, como en la de seguimiento $\left(\mathrm{F}_{(2,40)}=20.512, p<.000, \eta^{2}=.50\right)$. El test de Tuckey señala que en la fase de postratamiento los dos gru- 
pos que recibieron instrucción en estrategias de comprensión aventajaban al grupo de comparación, y que el grupo que recibió el entrenamiento en pequeño grupo aventajaba al grupo instruido en gran grupo. En la fase de seguimiento, sólo se mantienen las diferencias entre los dos grupos instruidos y el de comparación, no encontrándose diferencias entre los dos grupos que recibieron instrucción en estrategias de comprensión.

También se encuentran diferencias significativas entre los grupos en la medida de conocimiento de estrategias de la Bateria Surco, tanto en el postratamiento $\left(\mathrm{F}_{(2,40)}=13.165, p<\right.$ $\left..000, \eta^{2}=.39\right)$, como en el seguimiento $\left(\mathrm{F}_{(2,40)}=17.662, p<\right.$ $\left..000, \eta^{2}=.46\right)$. El test de Tuckey manifiesta que tanto en el postratamiento como en el seguimiento, los dos grupos que recibieron instrucción en estrategias de comprensión aventajaban al grupo de comparación, aunque no se observan diferencias entre ambas condiciones de instrucción.

En cuanto a la medida del uso de estrategias de comprensión de la Batería Surco, los ANOVAs entre grupos indican que se encuentran diferencias significativas en la fase de postratamiento $\left(\mathrm{F}_{(2,40)}=9.567, p<.000, \eta^{2}=.32\right)$, que se mantienen en la de seguimiento $\left(\mathrm{F}_{(2,40)}=24.199, p<.000, \eta^{2}=.54\right)$. Los resultados del test de Tuckey señalan que, tanto en el postratamiento como en el seguimiento, los dos grupos instruidos aventajan al de comparación, aunque el grupo instruido en pequeño grupo aventaja al resto de los dos grupos.
Por el contrario, respecto a la detección de fallos de comprensión, no se encuentran diferencias entre los grupos ni en el postratamiento, $\mathrm{F}_{(2,40)}=3.318, \mathrm{p}<.046, \eta^{2}=.14$, ni en el seguimiento, $\mathrm{F}_{(2,40)}=1.848, \mathrm{p}<.171, \eta^{2}=.08$.

\section{Resultados obtenidos en las medidas de generaliza- ción (ver Tabla 3)}

El ANOVA arrojó diferencias significativas entre grupos en la prueba de comprensión de la BEL, tanto en el postest $\left(F_{(2}\right.$, $\left.{ }_{40)}=24.810, p<.000, \eta^{2}=.55\right)$, como en el seguimiento $\left(F_{(2,}\right.$ $\left.{ }_{40)}=14.041, p<.000, \eta^{2}=.41\right)$. El test de Tuckey señala que, tanto en la fase de postratamiento como en la de seguimiento, los dos grupos que han recibido instrucción en estrategias aventajan al de comparación, aunque el grupo instruido en pequeño grupo tiene un rendimiento superior al grupo instruido en gran grupo.

Asimismo, en la habilidad para inferir el significado implícito se encuentran diferencias entre los grupos, tanto en postratamiento $\left(F_{(2,40)}=57.251, p<.000, \eta^{2}=.74\right)$, como en seguimiento $\left(F_{(2,40)}=37.999, p<.000, \eta^{2}=.65\right)$. En este caso el test de Tuckey señala que los chicos instruidos en PG aventajan a los otros dos (GG y GC), aunque los chicos del GG son superiores al GC en la fase de postratamiento. En la fase de seguimiento, únicamente se mantiene la superioridad de los dos grupos instruidos (PG y GG) sobre el GC.

Tabla 3. Medias y desviaciones típicas en las medidas de generalización.

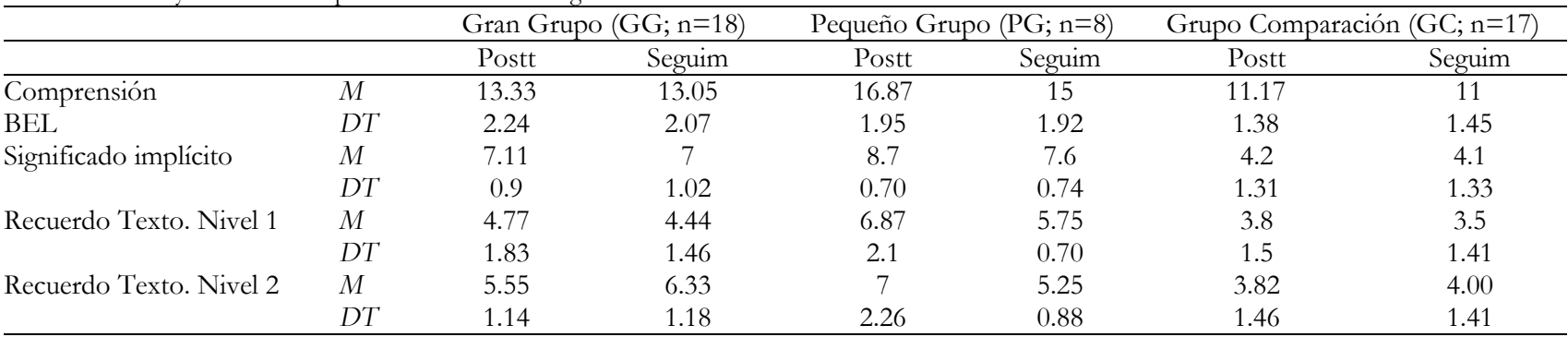

Por último, en relación a la prueba de recuerdo de ideas, los ANOVAs señalan diferencias significativas entre los grupos en el recuerdo de ideas importantes (nivel 1), para el postratamiento $\left(F_{(2,40)}=8.141, p<.001, \eta^{2}=.28\right)$, y seguimiento $\left(F_{(2,40)}=7.554, p<.002, \eta^{2}=.27\right)$, y para el recuerdo de ideas poco importantes (nivel 2), en postratamiento $\left(F_{(2,40)}=\right.$ $\left.15.540, p<.000, \eta^{2}=.43\right)$, y seguimiento $\left(F_{(2,40)}=12.968, p\right.$ $\left.<.000, \eta^{2}=.39\right)$. En relación al recuerdo de ideas importantes, el test de Tuckey señala que, tanto en el postratamiento como en el seguimiento, los chicos instruidos en PG aventajan a los otros dos (GG y GC), encontrándose un mejor rendimiento del GG con respecto al GC. En cuanto al recuerdo de ideas menos importantes, en la fase de postratamiento se observa la misma ejecución que en cuanto al recuerdo de ideas importantes. Sin embargo, en la fase de seguimiento, se mantiene la ventaja de los dos grupos instruidos en estrategias (PG y GG), aunque no se encuentran diferencias entre PG y GG.

\section{Conclusiones}

Los resultados de este estudio muestran claramente la eficacia de la ER para mejorar la comprensión lectora. Los sujetos de los dos grupos experimentales (GG y PG) mostraron ganancias significativas, tanto en las medidas de efectos específicos como en las de generalización, con respecto a los sujetos del grupo de comparación. Los efectos beneficiosos se observan en el posttest y se mantienen cuatro meses después en todas las medidas, a excepción de la medida de detección de fallos. La razón más plausible es que los textos naturales empleados presentaban pocas dificultades léxicas o ambigüedades sintácticas, con lo cual la clarificación de términos que no se entendían, así como de cualquier otra dificultad de comprensión fue una habilidad poco practicada. Así, nuestros resultados respaldan la eficacia de la ER aplicada por profesores en el aula ordinaria, coincidiendo con otros estudios previos (Alfassi, 1998, 2004; Brown y Palinc- 
sar, 1989; Hart y Speece, 1998; Hacker y Tenent, 2002; Kelly, Moore y Tuck, 1994; Mason, 2004; Rosenshine y Meister, 1994; Santiago, 2005; Soriano, Vidal-Abarca y Miranda, 1996; Soriano, Chebaani, Soriano y Descals, 2011).

En relación a nuestro segundo objetivo, comparar la eficacia de la instrucción en estrategias de comprensión mediante enseñanza recíproca desarrollada en pequeño grupo con la instrucción de dichas estrategias en gran grupo, los resultados muestran la superioridad del entrenamiento en pequeño grupo tanto en la fase de posttratamiento como en la de seguimiento. En concreto, el PG tuvo un rendimiento superior a los otros dos (GG y GC) en algunas de las medidas de efectos específicos (conocimientos de estrategias y actividades de comprensión lectora y uso de estrategias) y en todas las medidas de efectos de generalización (medida estandarizada, significado implícito y recuerdo de ideas) en consonancia a los resultados del meta-análisis realizado por Elbaum, Vaughn, Hughes y Moody (1999). Estos resultados posiblemente sean explicados porque la instrucción desarrollada en pequeños grupos de estudiantes proporciona un entorno en el que los estudiantes tenían más oportunidades de comunicar lo que saben, de practicar las estrategias y de recibir supervisión y retroalimentación del profesor y de otros estudiantes (Helf, Cooke, y Flowers, 2009).

Entre las limitaciones del presente estudio se encuentra la asignación no aleatoria de los niños a los grupos, pues li-

\section{Referencias}

Afflerbach, P. P. y Cho, B. Y. (2011). The classroom Assessment of reading. In M. L. Kamil, P. D. Pearson, E. B. Moje, \& P. P. Afflerbach (Eds.), Handbook of Reading Research. Volumen IV (pp. 487-514). New York: Routledge

Alfassi, M. (1998). Reading for meaning: The efficacy of reciprocal teaching in fostering reading comprehension in high school students in remedial reading classes. American Educational Research Journal, 35, 309-332.

Alfasssi, M. (2004). Reading to Learn: Effects of combined strategy instruction on high school students. The Journal of Educational Research, 97, 171184.

Alonso Tapia, J. y Carriedo, N. (1992). Evaluación de la supervisión y regulación de la comprensión. La Batería SURCO. En J. Alonso Tapia et al. (Eds.), Leer, comprender y pensar. Desarrollo de estrategias y técnicas de evaluación (pp. 11-57). Madrid: CIDE.

Alvermann, D. y Earle, J. (2003). Comprehension instruction. In A. P. Sweet \& C. Snow (Eds.), Rethinking reading comprehension (pp.12-30). New York: Guilford.

Berkeley, S., Scruggs, Th., y Mastropieri, M. (2010). Reading Comprehension Instruction for students with learning disabilities, 1995-2006: A metaanalysis. Remedial and Special Education, 31, 423-436.

Brown, A. L. y Campione, J. C. (1998). Designing a community of young learners: Theoretical and practical lessons. In N.M. Lambert and B. L. McCombs (Eds.), How students learn: Reforming schools through learner-centered education (pp. 153-186). Washington, DC: American Psychological Association.

Brown, A. L. y Palincsar, A. S. (1989). Guided, Cooperative Learning and Individual Knowledge Acquisition. In L. B. Resnick (Ed.), Knowing, Learning And Instruction. Hillsdale, N.J. L.E.A.

Brown, A. L. (1997). Transforming schools into communities of thinking and learning about serious matters. American Psychologist, 52, 399-413.

Canals, R. (1988). Pruebas Psicopedagógicas de Aprendizajes Instrumentales. Barcelona: Onda. mita el establecimiento de relaciones causales entre la instrucción y las ganancias en comprensión lectora.

Otra limitación, que afecta a la interpretación y a la generalización, es la falta de entrenamiento del grupo de comparación, aún en el supuesto caso de que hubiese podido recibir una enseñanza en comprensión de baja calidad (Sánchez, García, De Sixte, Castellano y Rosales, 2008). Sin embargo, las diferencias observadas entre los grupos experimentales y el de comparación pueden ser razonablemente atribuidas a la instrucción en estrategias recibida.

Los resultados de este estudio tienen un indudable interés para la enseñanza. Se confirma cómo nuestros alumnos, a través de la instrucción explícita de estrategias y de la ER realizada en las aulas ordinarias, y especialmente, en pequeños grupos, obtienen importantes beneficios, que se mantienen en el seguimiento, en la comprensión y aprendizaje a partir de textos, al menos con estudiantes de cuarto curso de enseñanza primaria. Dadas las diferencias evolutivas en estrategias de comprensión, es posible que con sujetos de otros niveles educativos los resultados fueran algo diferentes. Una respuesta a esta cuestión tendrá que ser abordada en ulteriores estudios.

Agradecimientos.- Este trabajo se ha desarrollado bajo un proyecto subvencionado por la Generalitat Valenciana, referencia GV2009-110, y por la Universidad de Valencia, referencia UV-AE-095730.

Cattell, R. B. y Cattell, A. K. S. (1950/1989). Test de Factor "g”. Escala 2. Madrid: TEA (Original publicado en 1950).

Cuetos, F., Rodríguez, B. y Ruano, E. (1996). Evaluación de los procesos lectores. PROLEC. Madrid: TEA.

De Vega, M., Cuetos, F., Domínguez, A. y Estévez, A. (1999). Diferencias individuales en lectura y comprensión. En M. de Vega y F. Cuetos (Eds.), Psicolingüistica del Español (pp. 231-271). Valladolid: Trotta.

Dole, J. A., Nokes, J. D., y Drits, D. (2009). Cognitive Strategy Instruction. In S.E. Israel y G.G. Duffy (Eds.), Handbook of Research on Reading Comprehension (pp. 347-372). New York: Routledge

Durkin, D. (1978-79). What classroom observations reveal about reading comprehension instruction. Reading Research Quaterly, 14, 481-533.

Elbaum, B., Vaughn, S., Hughes, M., y Moody, S. W. (1999). Grouping practices and reading outcomes for students with disabilities. Exceptional children, 65, 399- 415.

Gajria, M., Jitendra, A., Sood, Sh., y Sacks, G. (2007). Improving comprehension of expository text in students with LD: A research synthesis. Journal of Learning Disabilities, 40, 210-225.

Gilabert, R. (1990). La instrucción del significado por el contexto en la lectura comprensiva en el ciclo medio de la EGB (Tesis Doctoral no publicada). Universidad de Valencia.

Hacker, D. J. y Tenent, A. (2002). Implementing reciprocal teaching in the classroom: Overcoming obstacles and making modifications. Journal of Educational Psychology, 94, 699-718.

Hart, E. R. y Speece, D. L. (1998). Reciprocal teaching go to college: Effects for postsecondary students at risk for academic failure. Journal of Educational Psychology, 90, 670-681.

Helf, S., Cooke, N. L., y Flowers, C. P. (2009). Effects of two grouping conditions on students who are at risk for reading failure. Preventing School Failure, 53, 113-126.

Jitendra, A. K., Burgess, C., and Gajria, M. (2011). Cognitive strategy instruction for improving expository text comprehension of students with 
learning disabilities: The quality of Evidence. Exceptional Children, 77, 135-159.

Johnston, A. M., Barnes, M. A. y Desrochers, A. (2008). Reading Comprehension: Developmental Processes, Individual Differences, and Interventions. Canadian Psychology, 49, 125-132.

Kelly, M., Moore, D. W., y Tuck, B. F. (1994). Reciprocal Teaching in a regular primary classroom. Journal of Educational Research, 88, 53-61.

Leslie, L. y Caldwell, J. (2009). Formal and informal measures of reading comprehension. In S.E. Israel \& G. G. Duffy (Eds.), Handbook of Research on Reading Comprehension (pp. 403-427). New York: Routledge

López-Higes, R., Mayoral, J. A. y Villoria, C. (2002). Batería de Evaluación de la Lectura-BEL-. Madrid: Psymtec

Madariaga, J. M. y Martínez, E. (2010). The teaching of Reading Comprehension and metacomprehension strategies: A program implemented by teaching staff. Anales de Psicologia, 26, 112-122.

Malone, L. D. y Mastropieri, M. A. (1992). Reading comprehension instruction: Summarization and self-monitoring training for students with learning disabiliites. Exceptional Children, 58, 270-279.

Mason, L. H. (2004). Explicit self-regulated strategy development versus reciprocal questioning: Effects on expository reading comprehension among struggling readers. Journal of Educational Psychology, 96, 283-296.

Mayer, R. E. (1985). Structural analysis of science prose: can we increase problem-solving performance? In B. K. Britton \& J. B. Black (Eds.), Understanding expository text. Hillsdale, N. J.: LEA.

Miranda, A., Vidal-Abarca, E. y Villaescusa, I. (1997). Is attribution retraining necessary? Use of self-regulation procedures for enhancing the reading comprehension strategies of children with learning disabilities. Journal of Learning Disabilities, 30, 503-512.

Moody, S. W., Vaughn, S., y Schumm, J. S. (1997). Instructional grouping for reading. Remedial and Special Education, 18, 347-356.

National Reading Panel (2000). Teaching children to read: An evidence-based assessment of the scientific research literature on reading and its implications for reading instruction. Washington, Dc: National Institute of Child Health and Human development.

Ness, M. (2008). Supporting secondary readers: When teachers provide the "What", not the "How". American Secondary Education, 37, 80-95.

O'Connor, R. (2000). Increasing the intensivity of intervention in kindergarten and first grade. Learning Disabilities Research and Practice, 15, 43-54.

Palincsar, A., S. y Klenk, L. (1992). Fostering literacy learning in supportive contexts. Journal of Learning Disabilities, 25, 211-225.

Palincsar, A. S. y Brown, A. L. (1984). Reciprocal Teaching of Comprehension-Fostering and Comprehension-Monitoring Activities. Cognition and Instruction, 1, 117-175.

Pikulski, J.J. (1991). Grouping for literacy instruction: A need for thoughtful reconsideration. Florida Reading Quaterly, 27, 7-12.

Raphael, T.E., George, M., Weber, C., y Nies, A. (2009). Approaches to teaching reading comprehension. In S.E. Israel y G.G. Duffy (Eds.), Handbook of Research on Reading Comprehension (pp. 449-469). New York: Routledge

Reid, R. (1996). Research in Self-monitoring with students with learning disabilities: the present, the prospects, the pitfalls. Journal of Learning Disabilities, 29, 317-331.
Rosenshine, B. \& Meister, C.E. (1994). Reciprocal Teaching: A review of the research. Review of Educational Research, 64, 479-530.

Sánchez, E., García, J. R., De Sixte, R., Castellano, N. y Rosales, J. (2008). El análisis de la práctica educativa y las propuestas instruccionales: integración y enriquecimiento mutuo. Infancia y Aprendiraje, 31, 233-258.

Santiago, S. (2005). La comprensión de textos académicos en un contexto cooperativo guiado a través de la enseñanza recíproca. Revista Electrónica de Investigación Psicoeducativa y Psicopedagógica, 5, 77-96.

Sencibaugh, J. M. (2007). Meta-analysis of reading comprehension interventions for students with learning disabilities: Strategies and implications. Reading improvement, 44, 6-22.

Seymour, J. R. y Osana, H. P. (2003). Reciprocal teaching procedures and principles: Two teachers' developing understanding. Teaching and Teacher Education, 19, 325-344.

Shapiro, E. S., Durnan, S. L., Prost, E. E. y Skibitsky, T. (2002). Selfmonitoring procedures for children and adolescents. In M. R. Shinn, G. Stoner, \& H. Walker (Eds.), Interventions for academic and behavior problems II: Preventive and remedial approaches. Bethesda, MD: National Association of School Psychologist.

Solé, I. (1987). L'ensenyament de la comprensió lectora. Barcelona: CEAC.

Solé, I. y Teberosky, A. (2002). La enseñanza y el aprendizaje de la alfabetización: una perspectiva psicológica. En C. Coll, J. Palacios y A. Marchesi (Comp.), Desarrollo psicológico y educación. Vol. 2. Psicología de la Educación Escolar (pp. 461-657). Madrid: Alianza.

Solis, M., Ciullo, S., Vaughn, S., Pyle, N., Hassaram, B., y Leroux, A. (2011). Reading comprehension interventions for middle school students with learning disabilities: A synthesis of 30 years of research. Journal of Learning Disabilities. doi: 10.1177/0022219411402691

Soriano, M., Chebaani, F., Soriano, E., y Descals, A. (2011). Enseñanza Recíproca y Autoobservación del Uso de Estrategias: Efectos sobre la Comprensión de textos. Psicothema, 23, 38-43.

Soriano, M., Vidal-Abarca, E. y Miranda, A. (1996). Comparación de dos procedimientos de instrucción en comprensión y aprendizaje a partir de textos: instrucción directa y enseñanza recíproca. Infancia y Aprendizaje, 74, 57-65.

Spörer, N., Brunstein, J. C., y Kieschke, U. (2009). Improving students` reading comprehension skills: Effects of strategy instruction and reciprocal teaching. Learning and Instruction, 19, 272-286.

Stahl, K. A. D. (2009). Assessing the comprehension of young children. In S. E. Israel \& G. G. Duffy (Eds.), Handbook of Research on Reading Comprehension (pp. 428-448). New York: Routledge

Vaughn, S. \& Klingner, J. (2004). Teaching reading comprehension to students with learning disabilities. In C. A. Stone, E.R. Silliman, B. J. Ehren \& K. Apel (Eds.), Handbook of Language y Literacy. Development and Disorders (pp. 541-555). New York: Guildford.

Vidal-Abarca, E. (1989). La comprensión lectora de ideas principales en textos expositivos del ciclo medio de la EGB: Programa de instrucción y procesos cognitivos explicativos (Tesis Doctoral no publicada). Universidad de Valencia.

(Artículo recibido: 18-9-2012; revisado: 14-10-2012; aceptado: 16-10-2012) 